Classification

Physics Abstracts

$3115-31.20-33.10$

\title{
On Some Attempts to Generalize the Effective Hamiltonian Approach
}

\author{
J. L. Heully $\left({ }^{1}\right)$, S. Evangelisti $\left({ }^{2}\right)$ and Ph. Durand $\left({ }^{1}\right)$ \\ $\left({ }^{1}\right)$ Laboratoıre de Physique Quantique $\left({ }^{*}\right)$ Unıversité Paul Sabatıer, 118 route de Narbonne \\ 31062 Toulouse Cedex, France \\ $\left({ }^{2}\right)$ Dipartimento di Chimica Fisica e Inorganica, Universita di Bologna, Viale Risorgimento 4, \\ 40136 Bologna, Italy
}

(Recerved 18 July 1994, recerved in final form 10 October 1994, accepted 17 October 1994)

\begin{abstract}
A clear and sımple method to derive various intermediate Hamiltonians is proposed. Several intermediate Hamiltonians usable for various purposes are presented. The new intermediate Hamiltonians have the same properties as the traditional effective Hamiltonians but they should present better convergence properties, and should avold any intruder state problem
\end{abstract}

\section{Introduction}

Untll recently, the predominant correlation methods: many-body perturbation theory (MBPT) $[1,2]$ configuration-interaction (CI) [3] and coupled-cluster theories (CC) [4] were applied almost exclusively as single-reference based procedures. This is due in part to the increased computational complexity of the multireference-based procedures. For MBPT and CC one more reason is the requirement on the reference space to be complete yielding rather large reference spaces.

Although one-dimensional MBPT or CC have been shown to provide highly accurate energies and spectroscopic parameters, they are not universally applicable, particulary when exact or near degeneracies are encountered. They are usually insufficient for potential surfaces involving the dissociation of multiple bounds, and they are not ordinarily applicable to excited states other than the lowest ones of each symmetry. A rigourous treatment of such problems necessitates a multidimensional procedure. Quasidegenerate perturbation theory (QDPT) [5] has been receiving increasing attention in recent years. It provides the perturbational analogue of multireference configuration-interaction techniques (MRCI or CIPSI) [6] and it may be expected to provide faster convergence and more general applicability than single reference perturbation expansions. The earliest applications of QDPT $[7,8]$ (and its CC analogues) were all based on complete active spaces or complete model reference spaces. Such a reference space

$\left(^{*}\right)$ U.R.A. 505 du C.N.R.S. 
is a space where all configurations that can be generated by distributing the valence electrons among the valence orbitals are included. In many cases such a model space may be unnecessarily large and leads to computational difficulties. To face these difficulties incomplete model spaces have been advocated [9-13], where only the most strongly interacting configurations are included and the remaining ones are treated by perturbation. Such a reference space has a lower computational requirement and could reduce the intruder-state problem, when states originating from the model space interfere strongly with states of the complementary space.

The QDPT methods face a dilemma: very often a large model space is required to represent correctly the zeroth-order functions and at the same time a small model space is needed to avoid the intruder-state problem and save computational time Although the incomplete model space approach is obtaining several successes it leads to a reformulation of the theory and still requires a large computational effort.

A method which should solve the above problems has been proposed by Malrieu et al. [14] They claimed that the major difficulties with QDPT (and CC analogues) do not come from the size of the model space but from the condition imposed to the methods, vra the effective Hamiltonian, to give exact results for all the states of the model space. A much more reasonable approach is to pursue only a subset of true solutions, the other ones being approximated or neglected. This also leads to a new wave-operator, the so-called intermediate wave operator. Because some conditions on the effective Hamiltonian are relaxed the intermediate Hamiltonian is not uniquely defined and several different forms can be formulated.

One particular form has been used several times by our group with very promising results in difficult cases [15-17]. Another application with another intermediate Hamiltonian has been published [18]. Intermediate Hamiltonian schemes in Fock-space, based on the open-shell coupled-cluster formalism, have been proposed by Mukherjee [19] and Koch [20]. However, no exhaustive theoretical presentation of the intermediate Hamiltonian concept has been presented up to now. This is thus the aim of this article: to give a better theoretical foundation to the intermediate Hamiltonians and to present different forms which can be adapted for different problems. An effort is made to generalize the traditional QDPT to the concept, keeping as much as possible of the structure of the standard approach. Obviously, the problem is very complicated in its full generality, but it is found that new equations can be derived, which show great similarity with the standard equations. Only the formal aspects of the method will be discussed here. We expect to discuss their many-body realization in terms of diagrammatic expansions in future contributions.

Very recently we received a preprint by Meissner et al. [21] whose purpose is also to generalize the approach of Malrieu. Their method is based on effective Hamiltonians whereas ours is based on wave operators but the results are very similar. However, to avoid the intruder state problem Meissner et al. use a new iterative procedure which is not considered in this work. Our opinion is that this new scheme should give excellent convergence properties to the intermediate Hamiltonian perturbation theories.

\section{Basic Formalism}

2.1. The Model SPACE. - The concept of model space plays a central role in the theory of effective Hamiltonians. It is a finite $d$-dimensional subspace $S_{m}$ of the entire Hilbert space. Its orthogonal complement is the outer space $S_{m}^{\perp}$. The orthogonal projection operators associated with $S_{m}$ and $S_{m}^{\perp}$ are $P_{m}$ and $Q_{m}$, respectively:

$$
P_{m}=\sum_{m=1}^{d}|m\rangle\left\langle m\left|\quad Q_{m}=\sum_{\alpha}\right| \alpha\right\rangle\langle\alpha| \quad P_{m}+Q_{m}=1
$$


2.2. The Wave Operator and the Effective Hamiltonian. - A wave operator $\Omega$ is defined by

$$
\psi^{a}=\Omega \psi_{0}^{a} \quad(a=1,2 \ldots d)
$$

where $\psi^{a}$ are exact solutions of the Schrödinger equation

$$
H \psi^{a}=E^{a} \psi^{a}
$$

and $\psi_{0}^{a}$ are the corresponding zeroth-order functions, confined to the model space. The latter functions are eigenfunctions of an effective Hamiltonian

$$
H_{\mathrm{eff}} \psi_{0}^{a}=E^{a} \psi_{0}^{a}
$$

the eigenvalues being the corresponding exact energies. By combining equations (2), (3) and (4), the basic equation relating $H_{\text {eff }}$ and $\Omega$ can be derived

$$
H \Omega=\Omega H_{\text {eff }}
$$

Many effective Hamiltonians can be derived from equation (5). The simplest way is obtained by multiplying equation (5) by $P_{m}$ on the left and on the right

$$
H_{\mathrm{eff}}=\left(P_{m} \Omega P_{m}\right)^{-1} P_{m} H \Omega P_{m}
$$

If, as usually done, the intermediate normalization is adopted,

$$
P_{m} \Omega P_{m}=P_{m}
$$

equation (6) yields the usual Bloch effective Hamiltonian [22]

$$
H_{\mathrm{eff}}=P_{m} H \Omega P_{m}
$$

The intermediate normalization has the disadvantage, among many others [22, 23], that the effective Hamiltonian (8) is non-Hermitian and the corresponding zeroth-order wavefunctions

$$
\psi_{0}^{a}=P_{m} \psi^{a}
$$

are non-orthogonal.

This can be avoided by using other normalizations of the zeroth-order functions. However, the connection between different normalizations is rather well-documented $[24,25]$ and we shall only consider the intermediate normalization, thus working in Bloch's scheme.

With this normalization it can be shown that the wave-operator satisfies the generalized Bloch equation [26]:

$$
\left[\Omega, H_{0}\right] P_{m}=\left(V \Omega-\Omega P_{m} V \Omega\right) P_{m}
$$

where the Hamiltonian has been partitioned into a zeroth-order Hamiltonian $H_{0}$ and a perturbation $V$.

This equation can be conveniently used to generate a perturbative expansion of the operator $\Omega$. Expansion of both $\Omega$ and $H_{\text {eff }}$ can be found in Lindgren [27]. 


\section{Intermediate Hamiltonians and Intermediate Wave-Operators}

To the previous model space $S_{m}$ considered in Section 2, we now add a second subspace, the so called intermedzate space noted $S_{\imath}$, containing all the configurations strongly interacting with $S_{m}$ but which are of no direct interest. The sum of $S_{m}$ and $S_{\imath}$ defines our new model space of dimension $D$. We shall not pursue all the $D$ states emerging from the model space but only a restricted number $d<D$ states corresponding to the main model space $S_{m}$. We thus have two new projectors

$$
\mathbb{P}=P_{m}+P_{\imath} \quad \text { and } \quad \mathbb{Q}=1-\mathbb{P}
$$

$\mathbf{Q}$ is the projector on the new outer space. In order to keep the presentation clear, we shall follow the same scheme as in the preceding paragraph. An intermediate wave operator $R$, is defined such that

$$
\psi^{a}=R \bar{\psi}^{a} \quad(a=1,2 \ldots d)
$$

where the $\psi^{a}$ are $d$ exact solutions of the Schrödinger equation and $\bar{\psi}^{a}$ the corresponding zeroth-order functions belonging to the model space These functions are eigenfunctions of an intermediate Hamiltonian:

$$
H_{1 \mathrm{nt}} \bar{\psi}^{a}=E^{a} \bar{\psi}^{a} \quad a=1,2 \ldots d
$$

It should be noted that $H_{1 \mathrm{nt}}$ acts in a space of dimension $D>d$.

The $\bar{\psi}^{a}$ 's are defined, as in the normal QDPT, as the projections of the exact functions $\psi^{a}$ onto the model space.

$$
\overline{\psi^{a}}=\mathbb{P} \psi^{a} \quad a=1,2 \ldots d
$$

We are now looking for a relation similar to the relation (7) which was necessary for the derivation of $H_{\text {eff }}(8)$ and the equation for $\Omega(10)$. Unfortunately such a relation cannot be obtained directly because the $\bar{\psi}^{a}$ 's span a space of dimension $D$ and we have only $d$ relations to define them. By combining equations (12) and (14) we obtain the new general relation

$$
R \operatorname{PP} \psi^{a}=\psi^{a} \quad a=1,2 \ldots d,
$$

By using equation (2), the above equation can be transformed into the basic equation

$$
R \mathbb{P} \Omega P_{m}=\Omega P_{m}
$$

This operator equation is true when it acts on the right in the main model space with projector $P_{m}$. This relation can be rewritten as

$$
\begin{gathered}
R P_{m}+R P_{\imath} \Omega P_{m}=\Omega P_{m} \\
R P_{m}=\left(1-R P_{\imath}\right) \Omega P_{m}
\end{gathered}
$$

This relation is the fundamental relation that will be used in this paper for deriving intermediate Hamiltonians. The relation does not give all the possible intermediate Hamiltonians but, as we shall show, gives a large class of $H_{\mathrm{nt}}$ closely related to the normal QDPT. In equation (17), the right hand side $\Omega P_{m}$ is completely defined and thus if we give a prior a particular form to $R P_{\imath}, R P_{m}$ is completely defined by equation (17). We shall continue to define $R$ by projecting equation (16) on the left onto the various parts of $\mathbb{P}$ and then on $\mathbb{Q}$. The projection on $P_{m}$ gives

$$
P_{m} R P_{m}+P_{m} R P_{\imath} \Omega P_{m}=P_{m}
$$

and it seems convenient to choose $P_{m} R P_{2}=0$ in order to have

$$
P_{m} R P_{m}=P_{m}
$$


multiplying equation (16) on the left by $P_{\imath}$ gives

$$
P_{2} R P_{m}+P_{2} R P_{2} \Omega P_{m}=P_{2} \Omega P_{m}
$$

As in the previous case, it seems convenient to choose $P_{2} R P_{m}=0$ yielding

$$
P_{\imath} R P_{\imath}=P_{\imath}
$$

These two conditions can be gathered into the normalization condition

$$
\mathbb{P} R \mathbb{P}=\mathbb{P}
$$

In the normal QDPT, this relation is a direct consequence of relation (9) whereas in our case it is not compulsory. Multiplying both sides of equation (17) on the left by the projector on the outer space gives

$$
\mathrm{Q} R P_{m}+\mathbb{Q} R P_{\imath} \Omega P_{m}=\mathbb{Q} \Omega P_{m}
$$

where $\mathbb{Q} R P_{\imath}$ has to be defined in some way.

The choice of $\mathrm{Q} R P_{\imath}$ can be based on several arguments such as:

- computational difficulties;

- Hermiticity of the resulting $H_{\mathrm{nnt}}$;

- inclusion of some correlation to accelerate the convergence of $H_{\mathrm{unt}}$;

- inclusion of some correlation in order to have the non-exact intermediate energies $E^{2}$ and intermediate functions $R \bar{\psi}^{2}$ not too far from the exact ones...

There are also other arguments such as the size-consistency of $H_{\text {int }}$ but they are much more difficult to specify In the next paragraph we shall analyze and present different possible choices for $R$ and the resulting $H_{\text {int }}^{\prime} s$.

In the conventional QDPT the effective Hamiltonian is derived from the Bloch equation (5) which is a natural result of equations (2-4). Such an equation cannot be obtained in our case, at least if we want to keep equations defined on a projector $\mathbb{P}$ built from the unperturbed projectors $P_{m}$ and $P_{\imath}$.

Of course, an equation similar to equation (5) can be stated with the help of the perturbed projector

$$
\begin{gathered}
P_{M}=\sum_{a}\left|\overline{\psi^{a}}\right\rangle\left\langle\bar{\psi}^{\perp}\right| \\
H R P_{M}=R H_{\mathrm{nt}} P_{M} \\
a=1,2, \ldots d \quad P_{I}=\mathbb{P}-P_{M}
\end{gathered}
$$

The $\bar{\psi}^{a \perp}$ 's are the biorthogonal of the $\bar{\psi}_{a}^{\prime} s$. But the use of the unknown projectors $P_{M}$ and $P_{I}$ leads to a quite different formulation $[20,28]$. We shall postulate the existence of a pseudoBloch effective Hamiltonian

$$
H_{\mathrm{nt}}=\mathbb{P} H R \mathbb{P} \text {. }
$$

It is easy to see that this operator gives $d$ exact eigenvalues and $d$ eigenvectors which are the projections upon the model space of the exact vectors. On the other hand, nothing can be said about the other solutions. This effective Hamiltonian cannot be derived from the equation

$$
H R \mathbb{P}=R H_{\mathrm{int}} \mathbb{P}
$$

which is satisfied only when both its sides act on the $d$ projected exact states. 


\section{Different Choices of $R$}

We have seen that with various choices of $Q R P_{1}$ the pseudowave operator $R$ is solution of equations $(16,17)$. We shall look at different choices for $\mathbb{Q} R P_{\imath}$.

4.1. The Minimal Choice. - The most obvious and simplest choice for $\mathbf{Q} R P_{\imath}$ in equation (24) is $\mathbb{Q} R P_{\imath}=0$ giving

$$
\mathbf{Q} R \mathbb{P}=\mathbf{Q} \Omega P_{m}
$$

and thus since $\mathbb{P} R \mathbb{P}=\mathbb{P}$

$$
R=\mathbb{P}+\mathbb{Q} \Omega P_{m} .
$$

Multiplying equation (28) on the left then the same equation on the right by $H_{0}$ and then subtracting both equations and using equation (10) leads to

$$
\mathbb{Q}\left[R, H_{0}\right] P_{m}=\mathbb{Q}\left[\Omega, H_{0}\right] P_{m}=Q(V \Omega-\Omega V \Omega) P_{m}
$$

and

$$
H_{\mathrm{nt}}=\mathbb{P} H \mathbb{P}+\mathbb{P} V \mathbf{Q} \Omega P_{m}
$$

This Hamiltonian is strongly non-Hermitian because the perturbation acts only, on the right, in the main model space.

4.1.1. Perturbational Treatment - If this Intermediate Hamiltonian is built by perturbation care must be taken in the choice of the intermediate space. If we assume that a complete set of unperturbed orthonormal eigenfunctions $\left\{\phi^{a}\right\}$ and corresponding eigenvalues are available

$$
H_{0} \phi^{a}=E^{a} \phi^{a} \quad\left\langle\phi^{a} \mid \phi^{b}\right\rangle=\delta_{a, b}
$$

then the first and second-order expressions for the $R$ operator are

$$
\begin{gathered}
\mathbf{Q} R^{(1)} P_{m}=\frac{\mathbf{Q}}{E_{0}-H_{0}} V P_{m} \\
\mathbb{Q} R^{(2)} P_{m}=\frac{\mathbf{Q}}{E_{0}-H_{0}}\left[\frac{V \mathbf{Q} V}{E_{0}-H_{0}}+\frac{V P_{\imath} V}{E_{0}-H_{0}}-\frac{\mathbf{Q}}{E_{0}-H_{0}} V P_{m} V\right] P_{m}
\end{gathered}
$$

In the two above expressions it has been assumed that $H_{0}$ is degenerate in the main model space with all its eigenvalues equal to $E_{0}$. For the first-order term no special problem can occur but at the second-order some dangerous terms appear. The second term in the right hand side of equation (33b) gives large contributions if the intermediate configurations are strongly coupled to those of the main model space. This will make the perturbation expansion quite unreliable and thus this intermediate Hamiltonian should be used only to second-order.

4.1.2. Alternative Treatment. - In order to avoid the problem of the coupling between the main and the intermediate space, one should isolate the $P_{2} \Omega P_{m}$ part of $\Omega$. Let us rewrite

$$
\begin{aligned}
\Omega & =P_{m}+P_{\imath} \Omega P_{m}+X \\
& =\mathbb{P} \Omega P_{m}+X
\end{aligned}
$$

With this notation equation (30) can be written as

$$
\mathbb{Q}\left[R, H_{0}\right] P_{m}=\mathbb{Q}\left[\Omega, H_{0}\right] P_{m}=\mathbb{Q}(1-X) V(1+X) \mathbb{P} \Omega P_{m}
$$


The second term $\mathbb{P} \Omega P_{m}$, which could be divergent with a perturbational treatment, can be obtained by diagonalizing the intermediate Hamiltonian. With the $d$ exact zeroth-order eigenfunctions and their biorthogonals we can obtain $P_{\imath} \Omega P_{m}$ as

$$
P_{\imath} \Omega P_{m}=\sum_{a=1}^{d} P_{\imath}\left|\psi^{a}\right\rangle\left\langle\psi_{n}^{a \perp}\right| P_{m}
$$

In this way the most dangerous terms are treated to infinite order whereas the normal terms are included by usual perturbational methods and thus can be approximated at a certain order. Consider, for instance, a model space of single and double configuration interaction (SDCI) type where the fundamental state defines the main model space and all single and double excitations define the intermediate space. In such a case equation (30) gives

$$
\begin{aligned}
R^{0}=\mathbb{P} & H_{\mathrm{int}}^{(1)} & =\mathbb{P} V \mathbb{P}=\Delta E_{\mathrm{SDCI}} \\
R^{(1)}=0 & H_{\mathrm{int}}^{(2)} & =0 \\
R^{(2)}=\frac{\mathbb{Q}}{E_{O}-H_{0}} V \frac{P_{2}}{E_{0}-H_{0}} V P_{m} & H_{\mathrm{int}}^{(3)} & =\mathbb{P} V \frac{\mathbb{Q}}{E_{0}-H_{0}} V \frac{P_{2}}{E_{0}-H_{0}} V P_{m}
\end{aligned}
$$

where the projector $\mathbb{Q}$ is generated by triple and quadruple excitations and $P_{\imath}$ by single and double excitations. The fact that $R^{(1)}$ is zero shows that the SDCI energy is correct to thirdorder. The term $R^{(2)}$ shows that the SDCI wave function should be corrected at second order, the effective Hamiltonian at third order and hence the energy (diagonalized) at fourth order. By using equation (35) instead of equation (30) some higher orders can easily be included.

4.2. An Extended Treatment. - The previous choice of $\mathbb{Q} R P_{2}$ provided a very simple intermediate Hamiltonian but it could be more useful, in view of building more Hermitian $H_{\text {int }}^{\prime} s$ or having transferable effective interactions, to include some interactions between the intermediate states and the outer states, hence choosing $\mathbb{Q} R P_{2} \neq 0$. Equation (24) can be recast in commutator form. This is very convenient since it ensures the invariance of the wave-operator upon a translation in energy of the diagonal energies of $H_{0}$.

$$
\mathbb{Q}\left[R, H_{0}\right] P_{m}=\mathbb{Q}\left[\Omega, H_{0}\right] P_{m}-\mathbb{Q}\left[R P_{\imath} \Omega, H_{0}\right] P_{m}
$$

Once $\mathbb{Q} R P_{\imath}$ has been chosen, $\mathbb{Q} R P_{m}$ is determined in a unique way by equation (38). We shall present two ways of chosing the $\mathbb{Q} R P_{\imath}$ part. The first one will yield a new original intermediate Hamiltonian, the second one will generalize the approach proposed by Malrieu et al. in their first presentation of the intermediate Hamiltonian approach.

a) In the same spirit, as in the previous paragraph, we shall isolate the terms containing $P_{\imath} \Omega P_{m}$. For this purpose, by using equation (10), equation (38) can be written in the form

$$
\begin{aligned}
\mathbb{Q}\left[R, H_{0}\right] P_{m}= & \mathbb{Q}\left(V \bar{\Omega}-\bar{\Omega} P_{m} V \bar{\Omega}\right) P_{m}+\mathbb{Q}\left(V P_{\imath} \Omega-\bar{\Omega} P_{m} V P_{\imath} \Omega\right) P_{m} \\
& -\mathbb{Q} R P_{\imath}\left[\Omega, H_{0}\right] P_{m}-\mathbb{Q}\left[R P_{\imath}, H_{0}\right] \Omega P_{m}=A+B+C+D
\end{aligned}
$$

where

$$
\begin{aligned}
& \bar{\Omega}=\left(P_{m}+\mathbf{Q}\right) \Omega \\
A= & \mathbb{Q}\left(V \bar{\Omega}-\bar{\Omega} P_{m} V \bar{\Omega}\right) P_{m} \\
B= & \mathbb{Q}\left(V P_{\imath} \Omega-\bar{\Omega} P_{m} V P_{\imath} \Omega\right) P_{m} \\
C= & -\mathbf{Q} R P_{\imath}\left[\Omega, H_{0}\right] P_{m} \\
D= & -\mathbf{Q}\left[R P_{2}, H_{0}\right] \Omega P_{m}
\end{aligned}
$$


One particular solution is to put

$$
\mathbb{Q}\left[R, H_{0}\right] P_{m}=A+C
$$

which implies that the condition $B+D=0$ must also be satisfied. By this way, equation (39) is transformed into a system of two equations that will allow a unique definition of $\mathbf{Q} R P_{m}$ and QRP

$$
\begin{aligned}
\mathbb{Q}\left[R, H_{0}\right] P_{m} & =\mathbb{Q}\left(V \bar{\Omega}-\bar{\Omega} P_{m} V \bar{\Omega}\right) P_{m}-\mathbb{Q} R P_{\imath}\left[\Omega, H_{0}\right] P_{m} \\
\mathbb{Q}\left[R, H_{0}\right] P_{\imath} \Omega P_{m} & =\mathbb{Q}\left(V-\bar{\Omega} P_{m} V\right) P_{\imath} \Omega P_{m}
\end{aligned}
$$

The second equation (42) does not define $Q R P_{\imath}$ in a unique way. This can be done by transforming (42) into:

$$
\begin{aligned}
\mathbb{Q}\left[R, H_{0}\right] P_{m} & =\mathbb{Q}\left(V \bar{\Omega}-\bar{\Omega} P_{m} V \bar{\Omega} P_{m}-\mathbf{Q} R P_{\imath}\left[\Omega, H_{0}\right] P_{m}\right. \\
\mathbb{Q}\left[R, H_{0}\right] P_{\imath} & =\mathbb{Q}\left(V-\bar{\Omega} P_{m} V\right) P_{\imath}
\end{aligned}
$$

It can immediately be checked that (43) implies (42) by multiplying both sides of the second equation (42) on the right by $\Omega P_{m}$. Up to first order $R^{(1)}$ is equivalent to the wave operator given by the standard QDPT on the whole model space. This means that the second-order Hamiltonians are also equivalent and that information on the intermediate states begins to be lost at the third-order only.

Unfortunately, in the case of intruder states, these equations have only a formal interest because the second equation (43) will diverge in the same way as the QDPT would do. However, it is possible to define a new zeroth-order Hamiltonian such as

$$
\begin{aligned}
\tilde{H}_{0} & =H_{0}+P_{\imath} W P_{\imath} \\
& =\left(P_{m}+\mathbb{Q}\right) H_{0}+\sum_{\imath} \tilde{E}_{\imath}|\imath\rangle\langle\imath|
\end{aligned}
$$

where $W$ is a diagonal operator changing the energies of the intermediate space.

$$
\begin{aligned}
\mathbb{Q}\left[R, \tilde{H}_{0}\right] P_{m} & =\mathbb{Q}\left(V \bar{\Omega}-\bar{\Omega} P_{m} V \bar{\Omega}\right) P_{m}-\mathbb{Q} R P_{\imath}\left[\Omega, \tilde{H}_{0}\right] P_{m} \\
\mathbb{Q}\left[R, \tilde{H}_{0}\right] P_{\imath} & =\mathbb{Q}\left(V-\bar{\Omega} P_{m} V\right) P_{\imath}
\end{aligned}
$$

$V$ in equation (45) has not been modified by equation (44). It should be noted that $W$ does not act as a shifting operator because it does not change the partitionning of $H$ into $H_{0}$ and $V$ in the main model space. No particular care is required in the choice of $W$ and thus any $W$ avoiding the intruder states would be convenient. However, the final results for the intermediate states and only for them would depend strongly on this choice. Before looking at some particular cases we would like to summarize our approach.

i) we have defined a pseudo wave operator $R$ acting in a model space containing the main and intermediate states $[\mathrm{Eq} .(12)]$;

ii) a wave operator $\Omega$ has been introduced to discriminate between the main and intermediate states [Eq. (16)];

iii) The $R P_{2}$ part of $\mathrm{R}$ which is a priori arbitrary in the theory of intermediate Hamiltonians is chosen in such a way that it cancels the dangerous terms of equation (39) associated with the interaction between the intermediate states and the model state;

iv) a special shift on the intermediate states is introduced in order to avoid the intruder state problem in equations $(44,45)$. 
Further simplifications are obtained if the main model space is degenerate to the value $E_{0}$. In this case we have:

$$
\begin{aligned}
\mathbf{Q} R P_{m} & =\frac{\mathbf{Q}}{E_{0}-H_{0}}\left(\left(V \bar{\Omega}-\bar{\Omega} P_{m} V \bar{\Omega}\right) P_{m}-\sum_{\imath}\left(E_{0}-\tilde{E}_{\imath}\right) R|\imath\rangle\langle\imath| \Omega P_{m}\right) \\
\mathbb{Q}\left[R, \tilde{H}_{0}\right] P_{\imath} & =\mathbf{Q}\left(V-\bar{\Omega} P_{m} V\right) P_{\imath}
\end{aligned}
$$

If $W$ is chosen in such a way that all intermediate energies $E_{\imath}$ are moved to the energy $E_{0}$ of the main model space, expression (46) reduces to

$$
\begin{aligned}
\mathbb{Q} R P_{m} & =\frac{\mathbf{Q}}{E_{0}-H_{0}}\left(V \bar{\Omega}-\bar{\Omega} P_{m} V \bar{\Omega}\right) P_{m} \\
\mathbb{Q} R P_{\imath} & =\frac{\mathbf{Q}}{E_{0}-H_{0}}\left(V-\bar{\Omega} P_{m} V\right) P_{\imath}
\end{aligned}
$$

The intermediate Hamiltonian obtained from this wave operator is Hermitian up to the secondorder and should give much better results than the previous $H_{1 n t}$. Furthermore, if one is concerned with the construction of transferable effective Hamiltonians, such an intermediate Hamiltonian is well-suited because it ensures a continuity of the effective operators around avoided crossings.

If we take again the example of an SDCI model space with only the ground state in the main model space, the expansions of $R$ and $H_{1 n t}$ are the following:

$$
\begin{array}{rlrl}
R^{(0)}=\mathbb{P} & H_{\mathrm{int}}^{(1)} & =\mathbb{P} V \mathbb{P} \\
R^{(1)} P_{m}=0 ; & R^{(1)} P_{\imath}=\frac{\mathbb{Q}}{E_{0}-H_{0}} V P_{\imath} & H_{\mathrm{int}}^{(2)} & =\mathbb{P} V \frac{\mathbb{Q}}{E_{0}-H_{0}} V P_{\imath} \\
R^{(2)}=0 & H_{\mathrm{int}}^{(3)} & =0
\end{array}
$$

Already at second-order $H_{1 \mathrm{nt}}$ contains corrections but the next corrections appear only at the fourth-order. One can see in this example that this $H_{1 \mathrm{nt}}$ is totally different from the previous one. Though in both cases one includes corrections from the tri- and quadri-excitations, their numbers are quite different; $D$ in the previous case, $D(D-1)$ in this case. However, at first order, $H_{1 n t}$ is in both cases a very sparse matrix where most of the terms are zero. The corrections towards the second order will fill up the whole matrix representing $H_{\text {int }}$ which could be inconvenient.

b) In both schemes presented above, a major defect was that the convergence rate of $R$ was solely determined by $\Omega$. Knowing that standard QDPT has a very low (if any) convergence rate, this is a bad feature which should be avoided as much as possible. For this purpose we return to equation (38) and use equation (30).

$$
\mathbf{Q}\left[R, H_{0}\right] P_{m}=\mathbf{Q}\left(V \Omega-\Omega P_{m} V \Omega\right) P_{m}-\mathbf{Q}\left[R P_{\imath} \Omega, H_{0}\right] P_{m}
$$

In the first term on the right-hand side of equation (49) some terms $\Omega P_{m}$ can be replaced by $R P_{m}+R P_{2} \Omega P_{m}$ (see Eq. (17)). By this means two results can be obtained

- equation (49) becomes approximately an equation giving $R$ as a function of $R$; this should accelerate the convergence;

- the terms $P_{\imath} \Omega P_{m}$ will be pushed to the next order. 
We shall apply this method to the first and third $\Omega P_{m}$ in equation (49) and the other cases will be discussed later on.

This transformation yields

$$
\begin{aligned}
\mathbb{Q}\left[R, H_{0}\right] P_{m}= & \mathbb{Q}\left(V R-\Omega P_{m} V R\right) P_{m}+\mathbb{Q}\left(V R P_{\imath}-\Omega P_{m} V R P_{\imath}\right) \Omega P_{m} \\
& -\mathbb{Q} R P_{\imath}\left[\Omega, H_{0}\right] P_{m}-\mathbb{Q}\left[R P_{\imath}, H_{0}\right] \Omega P_{m}
\end{aligned}
$$

We can see that this equation, made up of four terms, has the same structure as equation (39) of the previous paragraph. We shall adopt the same method as previously by adding the first and third terms and by cancelling the second and fourth ones.

$$
\begin{aligned}
\mathbb{Q}\left[R, H_{0}\right] P_{m} & =\mathbb{Q}\left(V R-\Omega P_{m} V R\right) P_{m}-\mathbb{Q} R P_{\imath}\left[\Omega, H_{0}\right] P_{m} \\
\mathbb{Q}\left[R, H_{0}\right] P_{\imath} & =\mathbf{Q}\left(V R-\Omega P_{m} V R\right) P_{\imath}
\end{aligned}
$$

We have again a system of two equations giving $\mathbf{Q} R P_{m}$ and $\mathbf{Q} R P_{2}$ as a function of $R$. However the left-hand sides of these equations are still suffering from the intruder-state problem in the second equation (37) and hence the same shift method used in the previous paragraph has to be applied. The introduction of the transformed zero-order Hamiltonian of equation (44) allows us to pass from equation (51) to

$$
\begin{aligned}
\mathbf{Q}\left[R, \tilde{H}_{0}\right] P_{m} & =\mathbf{Q}\left(V R-\Omega P_{m} V R\right) P_{m}-R P_{\imath}\left[\Omega, \tilde{H}_{0}\right] P_{m} \\
\mathbb{Q}\left[R, \tilde{H}_{0}\right] P_{\imath} & =\mathbf{Q}\left(V R-\Omega P_{m} V R\right) P_{\imath}
\end{aligned}
$$

This system represents the most direct generalization of the intermediate Hamiltonian scheme presented by Malrieu et al. which assumed degeneracy within the main model space. It is valid for any model space, degenerate or not, in the main or intermediate model space. In the simplest case, when the main model space is degenerate, one can always choose the shift operator $W$ in such a way that all the intermediate states become degenerate with the main model space. In such a case $R P_{2}\left[\Omega, \tilde{H}_{0}\right] P_{m}=0$ and the two equations (52) can be merged into the following equation

$$
\mathbf{Q} R \mathbb{P}=\frac{\mathbb{Q}}{E_{0}-H_{0}}\left(V R-\Omega P_{m} V R\right) \mathbb{P}
$$

where $E_{0}$ is the energy in the main model space. This is the relation proposed in the original formulation of the theory by Malrieu et al. It was derived in a very different way and $E_{0}$ was presented as a free parameter whereas in our formulation $E_{0}$ is the energy of the main model space.

If we return to our SDCI example we have with this system of equations the following expansion

$$
\begin{aligned}
& R^{(0)}=\mathbb{P} \quad H^{(1)}=\mathbb{P} V \mathbb{P}=\Delta E_{\mathrm{SDCI}} \\
& R^{(1)}=\frac{\mathbf{Q}}{E_{0}-H_{0}} V P_{\imath} \quad H_{\mathrm{nt}}^{(2)}=\mathbb{P} V \frac{\mathbb{Q}}{E_{0}-H_{0}} V P_{\imath} \\
& R^{(2)}=\frac{\mathbf{Q}}{E_{0}-H_{0}} V \frac{\mathbf{Q}}{E_{0}-H_{0}} V P_{2} \quad H_{\mathrm{int}}^{(3)}=\mathbb{P} V \frac{\mathbf{Q}}{E_{0}-H_{0}} V \frac{\mathbf{Q}}{E_{0}-H_{0}} V P_{\imath}
\end{aligned}
$$

Comparison with equation (48) shows that this scheme gives a correction at third-order on the intermediate subspace of the intermediate Hamiltonian.

Let us return to the most general case, in which the transformation of $\Omega P_{m}$ in equation (49) can be applied to any $\Omega P_{m}$. This leads to several systems of equations for $\mathbf{Q} R P_{m}$ and $\mathbf{Q} R P_{\imath}$ 
looking like equations (51) or (53). All are equivalent at first order but differ in third or fourth order. When the main model space is degenerate one can use the very general equation

$$
\mathbb{Q} R \mathbb{P}=\frac{\mathbf{Q}}{E_{0}-H_{0}}(V X-Y V Z)
$$

where two of the three operators $X, Y$ and $Z$ can be either $\Omega$ or $R$.

\section{Conclusion}

This work presents several classes of effective operators which generalize the wave-operator and effective Hamiltonian of the traditional QDPT. We have kept the Bloch formalism as much as possible while the usual condition that all the solutions of the $H_{\text {eff }}$ have to be exact has been relaxed. Since there are several ways to get approximate solutions, there are many possibilities for constructing intermediate Hamiltonians. We have presented a systematic method to obtain intermediate Hamiltonians ranging from a very simple and economical one where almost no corrections are introduced for the intermediate states, to more sophisticated ones, one of them being the original $H_{1 n t}$ proposed by Malrieu et al. [14].

Concerning the application of the intermediate Hamiltonian to Quantum Chemistry one should admit that these intermediate Hamiltonians have only a formal or mathematical interest. On the one hand, the use of $H_{1 n t}$ to high perturbational order would imply the same amount of work as a simple diagonalization and surely will not be faster than a standard Davidson scheme. On the other hand the use of the second or third order $H_{\text {int }}$ in many-body problems would not result in any improvement because none of the presented $H_{\text {int }}$ have a correct size-consistency or are separable. Traditional QDPT was separable! However, Malrieu et al. have shown that, if one leaves the order by order concept and instead, for a given order, includes a little part of the next order, size-consistency could be restored. This will be published in a forthcoming paper.

\section{References}

[1] Kucharski S A, Noga J., Bartlett R J., J Chem. Phys. 90 (1989) 7282 and references therein.

[2] Iwata S., Freed K. F , J. Chem. Phys. 76 (1982) 5051,

Sheppard M. G , Freed K. F., Herman M. F, Yeager D. L, Chem Phys. Lett. 61 (1979) 577;

Freed K F., Sheppard M. G, J. Phys. Chem. 86 (1982) 2130.

[3] Shavitt T, In Methods of Electronic Structure Theory, H. F. Schaefer Ed. (Plenum, New York, 1977)

[4] Bartlett R 'J., J. Phys Chem. 93 (1989) 1697.

[5] Brandow R. H., Rev. Mod. Phys. 39 (1967) 771;

Lindgren I., $J$ Phys $B \mathbf{7}$ (1974) 2441.

[6] Brown F. B., Shavitt I., Shepard R., Chem. Phys. Lett 105 (1984) 363;

Buenker R. J., Peyerimhoff S. D., Theor. Chrm. Acta 35 (1974) 33;

Huron B., Malrieu J. P., Rancurel P., J. Chem. Phys 58 (1973) 5745.

[7] Sheppard M. G., Freed K. F., J. Chem. Phys. 72 (1980) 4158.

[8] Salomonson S, Lindgren I., Martensson A. M., Phys Scr. 21 (1980) 351.

[9] Hose G, Kaldor U., J. Phys. B 12 (1979) 3827.

[10] Jeziorski B, Monkhorst H.J., Phys. Rev. A 24 (1981) 1668. 
[11] Lindgren I., Phys Scr 32 (1985) 291, 611.

[12] Kutzelnigg W., Mukherjee D., Koch S, J Chem. Phys 87 (1987) 5092.

[13] Mukherjee D , Chem Phys. Lett 125 (1986) 207

[14] Malrieu J. P., Durand Ph, Daudey J. P, J. Phys. A 18 (1985) 809

[15] Evangelisti S, Daudey J P., Malrieu J. P., Phys Rev. A 35 (1987) 4930

[16] Heully J. L., Daudey J P., J. Chem Phys. 88 (1988) 1046

[17] Zaitsevskı A. V., Dementev A. I., Chem Phys. Lett 168 (1990) 589.

[18] Sanchez-Marın J., Malrieu J. P., Maynau D., Int. J Quantum Chem. 31 (1987) 903

[19] Mukherjee D, Int. J Quantum Chem. 20 (1986) 409.

[20] Koch S., Theor. Chrm Acta 81 (1991) 169.

[21] Meissner L, Nooijen M., Bartlett R. J., to be published.

[22] Bloch C, Nucl. Phys. 6 (1958) 329.

[23] Lindgren I, Mukherjee D., Phys Rept. 151 (1987) 93.

[24] Cave R. J., Davidson E. R., J. Chem Phys. 89 (1988) 6798

[25] Jorgensen F., Mol Phys. 29 (1975) 1137.

[26] Durand Ph., Malrieu J P., Ab initio Methods in Quantum Chemistry, K. P. Lawley Ed (Wiley \& Sons, 1987).

[27] Lindgren I., Morrıson J., Atomic Many-Body Theory, Sprınger Series in Chemıcal Physıcs, Vol. 13 (1982).

[28] Evangelısti S., Thèse d'Etat, Toulouse (1988);

Evangelısti S., Durand Ph., Heully J. L., Phys. Rev. A (1991) 43.

[29] Zaitsevskii A. V, Heully J. L, J. Phys. B 25 (1992) 603

[30] Malrieu J. P., Zaitsevski A., Heully J. L., to be publıshed 